Check for updates

Cite this: RSC Adv., 2017, 7, 55441

Received 20th October 2017

Accepted 30th November 2017

DOI: 10.1039/c7ra11622e

rsc.li/rsc-advances

\section{Testing a simple approach for theoretical evaluation of radiolysis products in extraction systems. A case of N,O-donor ligands for Am/Eu separation $\dagger$}

\author{
P. I. Matveev, (D) *a A. A. Mitrofanov, ${ }^{a}$ V. G. Petrov, (D) ${ }^{\text {a }}$ S. S. Zhokhov, ${ }^{a}$ A. A. Smirnova, ${ }^{a}$ \\ Yu. A. Ustynyuk iD a and S. N. Kalmykov ${ }^{a b}$ \\ In this paper, we studied the irradiation and ageing effect on the extraction properties of $N, N^{\prime}$-diethyl- $N, N^{\prime}$ - \\ di(p-hexylphenyl)-1,10-phenantroline-4,7-dichloro-2,9-dicarboxamide (1) and 2,5,9,12-tetra(n-hexyl)- \\ benzo[f]quinolino-[3,4-b]-[1,7]-naphthyridine-6,8(5H,9H)-dione (2) towards Am(III) and Eu(III). DFT \\ calculations were applied to estimate the probable ways of radiolysis for (1) and (2). Degradation \\ products were identified by high-resolution ESI-MS. The interaction of (1) and (2) with the products of \\ solvent radiolysis was established to be the most probable mechanism of ligand destruction.
}

\section{Introduction}

One of the main tasks for the modern nuclear industry is the development of new efficient technologies for the treatment of enormous accumulated volumes of high level radioactive waste (HLW). ${ }^{1}$ Final disposal of this type of waste requires construction of repositories which will be stable over very long periods of time, because the total radioactivity of HLW will decrease to the safe level of natural uranium no earlier than in 100000 years. Minor actinides (i.e. neptunium, americium and curium) have a major impact on the total long-term radioactivity of HLW although their content in the waste does not exceed $0.1 \%$. Isolation of minor actinides and their further burning in next generation nuclear reactors will allow a decrease in the mandatory safety period of a repository. For this, it is necessary not only to extract americium and curium from HLW, but also to separate them from lanthanides. The latter problem is particularly difficult, since the chemical properties of actinides and lanthanides are very close.

Solvent extraction is the most suitable method for separation of actinides and lanthanides at the industrial scale. There are a number of various organic ligands which are being tested for selective separation of minor actinides. ${ }^{2}$ Extractants and their organic solutions must have not only high efficiency and selectivity towards americium and curium but also high resistance to ionizing radiation. Radiolysis and its products can

${ }^{a}$ Lomonosov Moscow State University, Leninskie gory, 1 bld. 3, Moscow, 119991, Russia. E-mail: petr.i.matveev@gmail.com

${ }^{b} N R C$ Kurchatov Institute, Akademika Kurchatova pl. 1, 123182 Moscow, Russia

$\dagger$ Electronic supplementary information (ESI) available: Cartesian coordinates of the calculated structures and HR-ESI-MS spectra. See DOI: 10.1039/c7ra11622e result in destruction of extractants, formation of compounds with diverse extraction behavior, formation of third phase or sediments of different nature.

Earlier, the radiolytic stability of a number of extraction systems developed for both the group separation of $\mathrm{An}$ (III)/Ln(III) and their individual element's separation has been studied in details. ${ }^{3-7}$ The classical experimental approach for testing of the radiation stability is an external gamma irradiation of organic extractant solutions, subsequent extraction experiments and determination of qualitative and quantitative composition of radiolysis products. However only few studies were performed on the theoretical predictions of irradiation effects and the most radiation-stable ligands. In 2014, Koubský and Kalvoda ${ }^{8}$ had proposed a version of ab initio calculations for estimation of the radiolytic stability of ligands. It was shown that characteristics of molecular orbitals (MO) (e.g. difference between HOMO and LUMO) could be used as a descriptor of radiolytic stability. But such a descriptor is common for all possible reactions that can occur with the extractant. In the further studies ${ }^{9}$ of the same authors, the theoretical description of the reaction mechanisms for diglycolamide (DGA) during radiolysis was proposed.

One may propose two main routes of ligand destruction in gamma-irradiated "ligand in organic phase/nitric acid solution" system. First one is the direct bond destruction under irradiation and the second is the interaction of the ligand with radicals and ions formed by destruction of organic solvent, water, nitric acid and ligand itself. The second route is preferable for diluted $(<0.1$ M) extractants solutions., ${ }^{5,10}$ In this case, products of radical addition and/or substitution in initial ligand should form. That is why the prediction of ligand radiation stability must be based on our knowledge about the position of reaction centers and "strength" of bonds in initial ligand to evaluate probability of radical reactions. 
Here we propose relatively simple methods of population analysis (Mayers bond order and Fukui molecular orbital theory) in order to estimate main directions of radical attack under irradiation and to predict main radiolysis products. Fukui indices were used for example in ref. 11 for modelling of $\cdot \mathrm{OH}$ radical behavior in radiolysis process. However application of this method for complex systems with an appropriate accuracy needs much more computational resources.

Here we present the results of our study on radiolytic stability of $\quad N, N^{\prime}$-diethyl- $N, N^{\prime}-\operatorname{di}(p$-hexylphenyl)-1,10-phenantroline-4,7dichloro-2,9-dicarboxamide (1) and 2,5,9,12-tetra( $n$-hexyl)benzo[f]quinolino-[3,4- $b]$-[1,7]-naphthyridine-6,8(5H,9H)-dione (2) (Fig. 1). Although extraction properties of diamides of heterocyclic acids (pyridine-2,6-dicarboxylic, 2,2'-bipyridine6,6'-dicarboxylic and 1,10-phenantroline-2,9-dicarboxylic) towards actinides and lanthanides are well described the literature, ${ }^{\mathbf{1 2 - 1 4}}$ the data on their radiolytic stability is limited by those for the diamides of pyridine-2,6-dicarboxylic acid. ${ }^{15,16}$ The ligand (1) have earlier demonstrated high selectivity for separation of neighboring pairs of rare earth elements (REE), ${ }^{17}$ and the ligand (2) have demonstrated high selectivity for separation Am/Eu pair. ${ }^{18}$ A weakly polar mixture of 1-dodecanol and "Isopar-M" (mixture of branched hydrocarbons) was used as a solvent in both cases.

The aim of this work is to estimate the impact of different routes on the degradation of extractants (1) and (2) under external gamma-irradiation and to demonstrate in silico approach for the elucidation of radiolysis mechanism.

\section{Experimental}

\section{Reagents}

The ligands (1) and (2) were synthesized according to the procedures described in ref. 17 and 18, respectively. Purity of both compounds was confirmed by ${ }^{1} \mathrm{H}$ NMR, ${ }^{13} \mathrm{C}$ NMR and HRESI-MS.

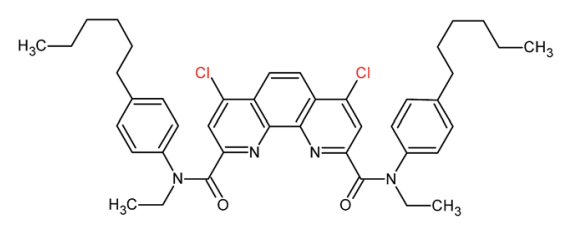

(1)

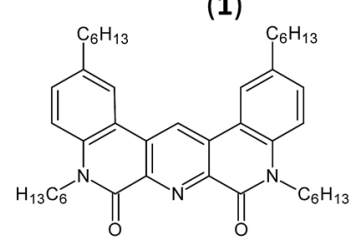

(2)

Fig. 1 Structure of $N, N^{\prime}$-diethyl- $N, N^{\prime}$-di(p-hexylphenyl)-1,10-phenantroline-4,7-dichloro-2,9-dicarboxamide (1) and 2,5,9,12-tetra( $n$ hexyl)-benzo[f]quinolino-[3,4-b]-[1,7]-naphthyridine-6,8(5H,9H)dione (2).
1-Dodecanol ("Khimreactiv") with purity 99\% and "Isopar-M" solvent ("Exxon Mobil Chemical") with purity of $98 \%$ were used without additional purification. "Isopar-M" solvent is a mixture of branched alkanes $\left(\mathrm{C}_{13}-\mathrm{C}_{14}\right)$ with a boiling point in the range of 207-250 ${ }^{\circ}$ C. Nitric acid ("Khimmed", a. g.) was used for the preparation of aqueous solutions with a given acidity by dilution with the deionized water Milli-Q (18 $\left.\mathrm{M} \Omega \mathrm{cm}^{-1}\right)$. Acetonitrile (HPLC gradient grade, Sigma Aldrich, USA) with formic acid (puriss. p.a., for HPLC, Fluka, Switzerland) were used as a diluent for electrospray ionization mass-spectrometry (ESI-MS) analysis. ${ }^{241} \mathrm{Am}$ and ${ }^{152} \mathrm{Eu}$ were purchased from ZAO "Isotope" (Russia).

\section{Irradiation and ageing of samples}

Irradiation of organic phases was performed using "GAMMA400" (Moscow State University) of cesium-137 gamma-source

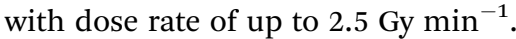

Concentration of ligands was $0.05 \mathrm{M}$. Irradiation was carried out in glass tubes. During irradiation, organic samples were kept in contact with water, 1 and $6 \mathrm{~mol} \mathrm{~L}^{-1}$ nitric acid, as well as pure organic with no aqueous phases have been irradiated for comparison. The volumes of the organic and aqueous phases were 3 milliliters. Mixed-phase systems were intensively shaken for 15 minutes before the irradiation for saturation and equilibration of the phases. During irradiation, no stirring was performed. Aliquots of organic phases were taken for subsequent solvent extraction experiments and for determination of the qualitative composition of radiolysis products by high resolution ESI-MS after accumulating the certain dose.

For the ageing experiments, the set of similar samples was kept in a dark place. Aliquots of organic phase were taken after 1, 2, 4 or 24 weeks. Time intervals in ageing experiments are corresponding to the periods of time necessary for the accumulation of doses of 25, 50, 100 and $600 \mathrm{kGy}$, respectively.

\section{Solvent extraction}

Solvent extraction experiments were carried out as follows. Half of milliliter of irradiated or aged organic phases and half of milliliter of aqueous phase containing $3 \mathrm{M}$ nitric acid, americium-241 (initial radioactivity $1.2 \mathrm{kBq} \mathrm{mL}^{-1}$ ) and europium-152 (initial radioactivity $2.0 \mathrm{kBq} \mathrm{mL}^{-1}$ ) were put in $1.5 \mathrm{~mL}$ polypropylene vial. The phases were stirred for 15 minutes on the vortex shaker at a temperature of $25.0 \pm 0.1^{\circ} \mathrm{C}$. After the phase contact, samples were centrifuged (5 minutes, $6000 \mathrm{rpm}$ ) and $0.35 \mathrm{~mL}$ of each phase was taken for determination of metal radioactivity. Content of ${ }^{241} \mathrm{Am}\left(E_{\gamma}=59.5 \mathrm{keV}\right)$ and ${ }^{152} \mathrm{Eu}\left(E_{\gamma}=121.8 \mathrm{keV}\right)$ was determined by gammaspectrometry using high-pure germanium detector GR 3818 (Canberra Ind.). The relative error of each measurement did not exceed $5 \%$. The distribution coefficients $(D)$ of metals were calculated as the ratio of the counting rate in the organic and aqueous phase, the separation factors $(\mathrm{SF})$ were calculated as the ratio of the distribution coefficients.

\section{Mass spectrometry analysis}

The aliquots of organic phases contained ligands after radiolysis or ageing were diluted with acetonitrile (HPLC gradient grade, 
Sigma-Aldrich, USA) containing 1\% formic acid (puriss. p.a., for HPLC, Fluka, Switzerland) until the final concentration of $10^{-5} \mathrm{M}$. Experiments were carried out with Orbitrap Elite mass spectrometer (Thermo Fisher Scientific GmbH, Bremen, Germany) with electrospray ionization source. Samples were introduced into the ion source using a syringe pump at flow rate $3 \mu \mathrm{L} \mathrm{min}{ }^{-1}$.

Mass spectra were acquired in a positive ionization mode. Sheath gas flow rates were from 10 to 25 arbitrary units, auxiliary and sweep gas flow rate was set to zero, spray voltage $3.5 \mathrm{kV}$, capillary temperature $275^{\circ} \mathrm{C}$, S-Lens rf level $60 \%$. Masses were analyzed in Orbitrap mass analyzer with 480000 resolving power. Fragmentation was done by collision induced dissociation (CID) at normalized collision energy (NCE) 40. All spectra were registered continuously during 1 minute, then averaged. Results were processed using Thermo XCalibur 3.0 software (Thermo Fisher Scientific Inc.).

Mass spectrometry analysis was performed for the samples of the ligand (1) irradiated to the doses of 25 and $200 \mathrm{kGy}$, as well as for the samples after ageing for 6 months. In the case of the ligand (2) accumulated doses were 100, 200 and $300 \mathrm{kGy}$.

\section{Computational details}

We performed geometry optimization of the ligands (1) and (2) and the solvent molecules in three steps: (1) conformational analysis with semiempirical Hamiltonian PM7 (ref. 19) implemented in MOPAC2016 package, ${ }^{20}$ (2) preliminary geometry optimization at DFT level of theory with hybrid functional B3LYP ${ }^{21,22}$ and 6-31G basis set ${ }^{23}$ in ORCA package ${ }^{24}$ and (3) geometry and electron structure refinement for further analysis at DFT level of theory with Grimme dispersion correction D3 (D3-B3LYP/def2TZVPP $^{21,22,25}$ ). In order to estimate radiation stability we used two methods of the electron structure analysis: Mayer population analysis $^{26-28}$ and Fukui frontier molecular orbitals theory. ${ }^{29,30}$

Mayer bond order (MBO) calculation is based on Mulliken population analysis and expression of molecule electron structure as a system of two-centered bonds. Bond order for A and B atoms is defined as:

$$
B_{\mathrm{AB}}=\sum_{\mu}^{\mathrm{A}} \sum_{\nu}^{\mathrm{B}}(P \mathrm{~S})_{\mu \nu}(P \mathrm{~S})_{\nu \mu}+(R \mathrm{~S})_{\mu \nu}(R \mathrm{~S})_{\nu \mu},
$$

where $P$ stands for electron density, and $R$ spin-density matrices. MBO calculations were performed in ORCA package.

Condensed Fukui indices calculation is based on frontier molecular orbitals (HOMO, HOMO - 1 and LUMO) and computation of electron density distribution for neutral molecule and \pm 1 ions with geometry optimized for neutral molecule. Radical susceptibility $(R)$, electrophilicity $(E)$, and nucleophilicity $(N)$ are defined as:

$$
\begin{gathered}
R(\mathrm{~A})=\frac{1}{2}\left(P_{\mathrm{A}}(N+1)-P_{\mathrm{A}}(N-1)\right) \\
E(\mathrm{~A})=P_{\mathrm{A}}(N+1)-P_{\mathrm{A}}(N) \\
N(\mathrm{~A})=P_{\mathrm{A}}(N)-P_{\mathrm{A}}(N-1)
\end{gathered}
$$

where $P$ stands for A atom occupancy. Electron density analysis were performed using natural atomic orbitals (NAO) theory ${ }^{31}$ in JANPA package. ${ }^{32}$

Simultaneous use of two mentioned methods allows us either to determine potential 'weak' bonds, or predict direction of further radical reactions.

All images were obtained with GABEDIT package. ${ }^{33}$

\section{Results and discussion}

\section{Solvent extraction experiments}

$N, N^{\prime}$-Diethyl- $N, N^{\prime}$-di( $p$-hexylphenyl)-1,10-phenantroline-4,7dichloro-2,9-dicarboxamide (1). Extraction experiments were performed on the intact, irradiated, and aged organic phases. The results expressed as $D(\mathrm{Am})$ and $\mathrm{SF}_{\mathrm{Am} / \mathrm{Eu}}$ values are presented on Fig. 2 and Table 1, respectively. $D(\mathrm{Am})$ value strongly depends on the absorbed dose and irradiation conditions. For the samples that had no contact with aqueous phases, one could observe a linear drop of $D(\mathrm{Am})$ with increasing dose value in semi-logarithmic coordinates. For the samples which were in contact with water, a slight growth of both distribution coefficient and Am/Eu separation factor was observed at 25 and 50 kGy doses. This effect can be associated with formation of compounds with better extraction ability under these conditions, which are degraded upon further irradiation. At $100 \mathrm{kGy}$ and higher, the extraction ability linearly dropped in a fashion similar to the samples that did not contact with aqueous phase.

Quite different dependence was observed when organic solutions of (1) were irradiated in contact with nitric acid solutions. A sharp decrease in the extraction ability and selectivity was seen already at the relatively low dose of $25 \mathrm{kGy}$, and then much smoother decrease in the americium distribution coefficient upon further increase of the absorbed dose.

Much smaller effect on the extraction properties was observed for the samples aged without contact with aqueous phase or in contact with pure water. These samples lost only $c a$. $25 \%$ of their initial selectivity after 6 months of ageing. At the same time, the ageing of (1) solutions in an organic solvent in contact with nitric acid led to a rapid decrease in the extraction properties. Upon contact with $6 \mathrm{M}$ acid, the complete loss of extraction properties occurred within 1 month. Thus, in these conditions the main process is acid-induced ligand destruction.

2,5,9,12-Tetra(n-hexyl)-benzo[f]quinolino-[3,4-b]-[1,7]-naphthyridine-6,8(5H,9H)-dione (2). Ligand (2) demonstrated

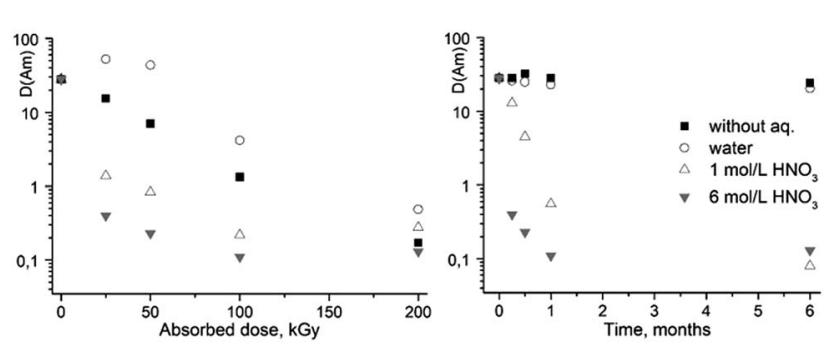

Fig. 2 The dependence of $D$ values for Am(III) on absorbed dose (left) and on the ageing time of the solutions (right). The extractant is $0.05 \mathrm{M}$ ligand (1) solution in dodecanol/"Isopar-M". 
Table 1 The dependence of Am/Eu SF for organic phase on irradiation dose or ageing time. The extractant was $0.05 \mathrm{M}$ ligand (1) in dodecanol/ "Isopar-M"

\begin{tabular}{|c|c|c|c|c|c|c|c|c|c|}
\hline Irradiation/ageing conditions & 0 & 25 & 50 & 100 & 200 & 0.25 & 0.5 & 1 & 6 \\
\hline Water & 85 & 115 & 110 & 14 & 13 & 87 & 83 & 77 & 65 \\
\hline $1 \mathrm{M} \mathrm{HNO}_{3}$ & 85 & 15 & 8 & 13 & 10 & 52 & 35 & 7 & 5 \\
\hline $6 \mathrm{M} \mathrm{HNO}_{3}$ & 85 & 12 & 9 & 10 & 10 & 8 & 5 & 5 & 3 \\
\hline
\end{tabular}

a completely different and rather unique dependence of extraction properties on irradiation and ageing (Fig. 3 and Table 2). The samples contacted with nitric acid solutions fully kept their extraction properties after irradiation up to $500 \mathrm{kGy}$, while for the samples contacted with pure water or even without contact with aqueous phase the $D$ values for americium dropped down to a value less than 1 at the dose of $300 \mathrm{kGy}$. A similar protective effect of nitric acid under external gamma-irradiation had been previously observed for some other extractants, such as 2,6-bis-[5(2,2-dimethyl-propyl)-1 $H$-pyrazol-3-yl]-pyridine in 1-octanol ${ }^{34}$ and carbamoylphosphine oxide ${ }^{35}$ but it has still not been explained from the view point of preferential radical reaction routes.

In the ageing experiment, it was shown that contact of ligand (2) with aqueous phases without irradiation does not affect its extraction properties for up to 6 months.

Thus, from the results of solvent extraction experiments we may conclude that the ligand (1) is unstable in contact with acidic solutions. Even for the systems without contact with nitric acid solutions the value of distribution coefficient of Am(III) was less than 1 at absorbed dose of about $150 \mathrm{kGy}$. On the contrary, the presence of nitric acid demonstrates a protective effect on (2) radiation stability. No changes in extraction properties were observed up to at least $500 \mathrm{kGy}$ in the systems equilibrated with 1 and $6 \mathrm{M}$ nitric acid solution. Analysis of the radiolysis products should provide necessary information for understanding of the processes leading to different behavior of these ligands under irradiation in contact with nitric acid.

\section{ESI-MS analysis of ageing products}

Ligand (1). Samples of organic phases aged during six months were analyzed by high-resolution ESI-MS. All the identified compounds are listed in Table 3. In the case of the samples contacted with pure water, the most intense was a peak
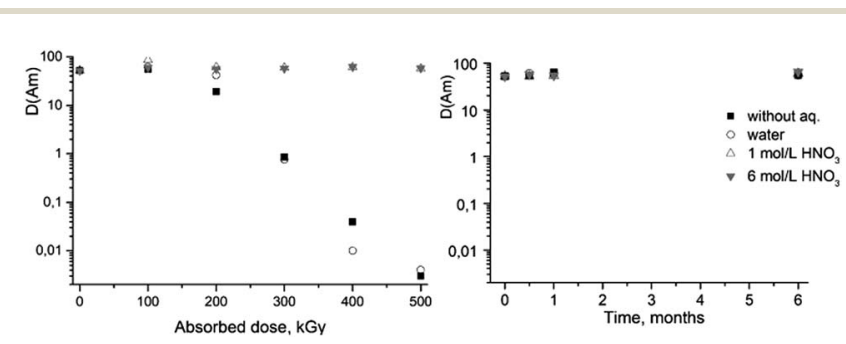

Fig. 3 The dependence of $D$ values for Am(III) on absorbed dose (left) and on the ageing time of the solutions (right). The extractant is $0.05 \mathrm{M}$ ligand (2) solution in dodecanol/"Isopar-M".
Table 2 The dependence of Am/Eu separation factors for organic phase on irradiation dose or ageing time. The extractant was $0.05 \mathrm{M}$ ligand (2) in dodecanol/"Isopar-M"

\begin{tabular}{|c|c|c|c|c|c|c|c|c|c|}
\hline \multirow[b]{2}{*}{ Irradiation/ageing conditions } & \multicolumn{6}{|c|}{ Absorbed dose, kGy } & \multicolumn{3}{|c|}{$\begin{array}{l}\text { Ageing } \\
\text { time, } \\
\text { months }\end{array}$} \\
\hline & 0 & 100 & 200 & 300 & 400 & 500 & 0.5 & 1 & 6 \\
\hline Without aqueous phase & 10 & 7 & 8 & 3 & 2 & 2 & 7 & 9 & \\
\hline Water & 10 & 8 & 4 & 4 & 2 & 2 & 7 & 7 & 6 \\
\hline $1 \mathrm{M} \mathrm{HNO}_{3}$ & 10 & 11 & 10 & 7 & 8 & 8 & 10 & 8 & 7 \\
\hline $6 \mathrm{M} \mathrm{HNO}_{3}$ & 10 & 11 & 8 & 10 & 9 & 8 & 8 & 7 & 7 \\
\hline
\end{tabular}

Table 3 Ageing products of the ligand (1) determined by ESI-MS

\begin{tabular}{|c|c|c|c|}
\hline No. & $\begin{array}{l}\text { Peak } \\
m / z\end{array}$ & $\begin{array}{l}\text { Molecular formula } \\
\text { and monoisotopic } \\
\text { mass }\end{array}$ & The proposed structure \\
\hline I & 711.32 & $\begin{array}{l}\mathrm{C}_{42} \mathrm{H}_{48} \mathrm{Cl}_{2} \mathrm{~N}_{4} \mathrm{O}_{2} \\
710.3154\end{array}$ & \\
\hline II & 693.36 & $\begin{array}{l}\mathrm{C}_{42} \mathrm{H}_{49} \mathrm{ClN}_{4} \mathrm{O}_{3}, \\
692.3493\end{array}$ & \\
\hline III & 738.34 & $\begin{array}{l}\mathrm{C}_{42} \mathrm{H}_{48} \mathrm{ClN}_{5} \mathrm{O}_{5}, \\
737.3344\end{array}$ & \\
\hline IV & 674.37 & $\begin{array}{l}\mathrm{C}_{40} \mathrm{H}_{52} \mathrm{ClN}_{3} \mathrm{O}_{4}, \\
673.3646\end{array}$ & \\
\hline
\end{tabular}

$\mathrm{R} 1=\mathrm{Cl}, \mathrm{R} 2=\mathrm{OH}$ or $\mathrm{R} 1=\mathrm{OH}, \mathrm{R} 2=\mathrm{Cl}$

at $m / z 711.32$ corresponding to a protonated ion of the initial ligand (I), as an impurity, there was an additional peak at $\mathrm{m} / \mathrm{z}$ 693.36 corresponding to the product of substitution of one chlorine atom by the hydroxyl group (II). This peak was the most intense in the samples contacted with 1 or $6 \mathrm{M}$ nitric acid, while a peak of the initial ligand (I) was not detectable at all. In these 
samples, there were also relatively small peaks at $m / z 738.34$ (III) and 674.37 (IV). Compound III appears to be a nitration product of II, while IV is presumably an alkoxylated hydrolysis product of ligand (1). In the case of contact with $1 \mathrm{M}$ nitric acid solution, the intensity of peak IV was c.a. 5\% of the main peak (II) intensity, and in the case of $6 \mathrm{M}$ nitric acid solution its intensity reached c.a. $35 \%$ of (II).

Thus, a contact of organic phase with water results in a slow substitution of one chlorine atom in the ligand (1) by hydroxyl group. The process is accelerated in the presence of nitric acid. As far as amides of heterocyclic acids can extract nitric acid, ${ }^{12}$ we can assume that protonation of the phenantroline nitrogen atoms leads to the activation of chlorine atoms in the reactions of nucleophilic aromatic substitution. As seen from the extraction data (Fig. 2), compound II demonstrates weak extraction properties $(D(\mathrm{Am})=0.1-0.3)$.

We did not perform the analysis of ageing products for ligand (2), because no changes of its extraction properties were observed during its ageing.

\section{ESI-MS analysis of radiolysis products}

Ligand (1). The peaks observed in ESI-MS spectra of ligand (1) after its radiolysis under different conditions, as well as the molecular formulas of radiolysis products and their putative chemical structures are summarized in Table 4 . The abundances of these peaks in individual spectra of samples irradiated under certain conditions are listed in Table 5 .

Under radiolysis conditions, more different products were formed from ligand (1) than we could observe under ageing conditions at the absence of irradiation. Nevertheless, the peak of compound II, which had been already detected in the aged samples, was the most intense peak almost in all the irradiated analyzed samples, including samples irradiated without aqueous phases. According to the work by Stephenson and Stuart, ${ }^{36}$ the water solubility in 1-dodecanol is up to 2.5 weight percent (up to $1.4 \mathrm{M}$ ), that is enough to replace chlorine atoms at the ligand content of $0.05 \mathrm{M}$. Since 1-dodecanol was used without additional drying, the content of dissolved water in the solvent could be a source of hydroxy radicals.

Table 4 The radiolysis products of the ligand (1) identified by ESI-MS

The proposed structure

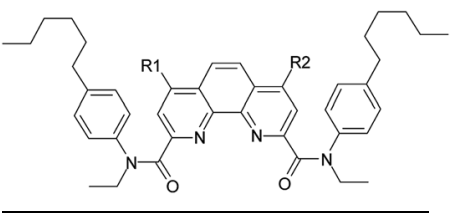

\begin{tabular}{lrl} 
No. & Peak $m / z$ & Molecular formula and \\
\hline I & 711.32 & \\
II & 693.36 & $\mathrm{C}_{42} \mathrm{H}_{48} \mathrm{Cl}_{2} \mathrm{~N}_{4} \mathrm{O}_{2}, 710.315$ \\
V & 861.54 & $\mathrm{C}_{42} \mathrm{H}_{49} \mathrm{ClN}_{4} \mathrm{O}_{3}, 692.3493$ \\
VI & 1011.76 & $\mathrm{C}_{54} \mathrm{H}_{73} \mathrm{ClN}_{4} \mathrm{O}_{3}, 860.5371$ \\
VII & 843.58 & $\mathrm{C}_{66} \mathrm{H}_{98} \mathrm{~N}_{4} \mathrm{O}_{4}, 1010.7588$ \\
VII & $\mathrm{C}_{54} \mathrm{H}_{74} \mathrm{~N}_{4} \mathrm{O}_{4}, 842.5710$
\end{tabular}

$\begin{array}{ll}\mathrm{R} 1 & \mathrm{R} 2 \\ \mathrm{Cl} & \mathrm{Cl} \\ \mathrm{Cl} & \mathrm{OH} \\ \mathrm{Cl} & \mathrm{C}_{12} \mathrm{H}_{25} \mathrm{O} \\ \mathrm{C}_{12} \mathrm{H}_{25} \mathrm{O} & \mathrm{C}_{12} \mathrm{H}_{25} \mathrm{O}\end{array}$

$\mathrm{OH} \quad \mathrm{C}_{12} \mathrm{H}_{25} \mathrm{O}$

VIII

206.19

$\mathrm{C}_{14} \mathrm{H}_{23} \mathrm{~N}, 205.1830$

IX

235.18

$\mathrm{C}_{14} \mathrm{H}_{23} \mathrm{~N}_{2} \mathrm{O}, 235.1810$

IV $674.37 \quad \mathrm{C}_{40} \mathrm{H}_{52} \mathrm{ClN}_{3} \mathrm{O}_{4}, 673.3646$

IV $674.37 \quad \mathrm{C}_{40} \mathrm{H}_{52} \mathrm{ClN}_{3} \mathrm{O}_{4}, 673.3646$

IV $674.37 \quad \mathrm{C}_{40} \mathrm{H}_{52} \mathrm{ClN}_{3} \mathrm{O}_{4}, 673.3646$
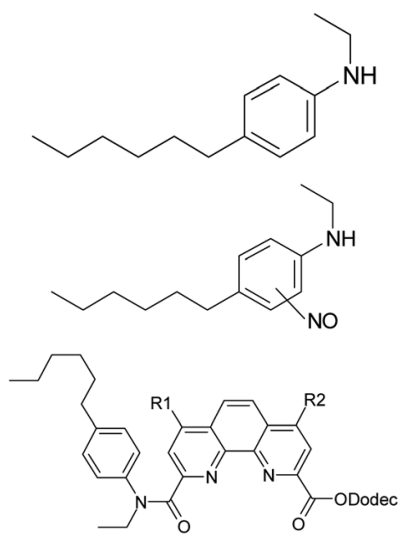

$$
\begin{gathered}
\mathrm{R}_{1}=\mathrm{Cl}, \mathrm{R}_{2}=\mathrm{OH} \text { or } \\
\mathrm{R}_{1}=\mathrm{OH}, \mathrm{R}_{2}=\mathrm{Cl}
\end{gathered}
$$

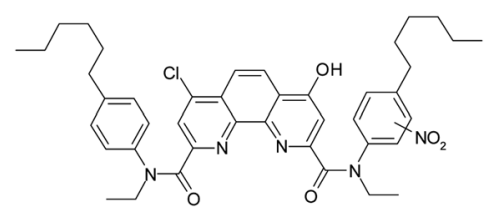


Table 5 Compounds formed from ligand (1) by irradiation under certain conditions. Compounds in bold are corresponding to the peaks of maximal intensities. The relative intensities of other peaks are shown in brackets

\begin{tabular}{llll}
\hline & Absorbed dose, kGy & \multirow{2}{*}{ Ageing time, months } \\
\cline { 2 - 3 } Irradiation/ageing conditions & 25 & 200 & 6 \\
\hline Without aqueous phase & II, V (55\%), VI (15\%), VIII $(20 \%)$ & VIII, II (25\%) & I \\
Water & II, V (70\%), I (60\%), VIII (15\%) & VIII, II (27\%) & I, II (33\%) \\
$1 \mathrm{M} \mathrm{HNO}_{3}$ & II, V $(15 \%)$, VIII (8\%), I (5\%) & II, III (10\%) & II, III (5\%), IV (5\%) \\
$6 \mathrm{M} \mathrm{HNO}_{3}$ & II, IV (20\%), III (1\%) & II, IV (40\%), III (5\%) & II, IV (35\%), III (5\%)
\end{tabular}

The initial ligand (I) was identified only in two samples that was irradiated at $25 \mathrm{kGy}$ in contact with water or with $1 \mathrm{M}$ nitric acid, but the last case its peak was rather small. Thus, the process of chlorine substitution by hydroxyl was accelerated by irradiation due to formation of ${ }^{\circ} \mathrm{OH}$ radicals from water molecules. We can propose the same behavior for all diamides of 4,7dichloro-1,10-phenantraline-2,9-dicarboxylic acid.

As it was shown in the Extraction experiments section, the samples of ligand (1) which were not equilibrated with $\mathrm{HNO}_{3}$ solutions demonstrated good extraction properties after irradiation by doses up to $25 \mathrm{kGy}$. For the sample irradiated in contact with water, a distribution coefficient of americium was even higher than for the initial ligand. These organic samples contained the compound $\mathbf{V}$, a substitution product of one chlorine atom by $\mathrm{C}_{12} \mathrm{H}_{25} \mathrm{O}$. It is well known ${ }^{34}$ that aliphatic alcohols turn into alpha-radicals during irradiation and these radicals react with dissolved substances. So, we can assume that compound $\mathbf{V}$ may have better extraction efficiency and selectivity than the initial ligand (1).

In addition to compound $\mathbf{V}$, a peak of 4-hexylphenylethylamine (VIII) was detected in several samples. When irradiated by a small dose of $25 \mathrm{kGy}$, this compound was a minor component, but at $200 \mathrm{kGy}$ its peak became the most intense in the samples of organic phases not equilibrated with nitric acid solution. It should be noted that the second product of amide bond hydrolysis (4-chloro-6-hydroxy-1,10-phenanthroline-2,9dicarboxylic acid or its monoamide) was not identified in any registered spectra. That was probably due to low solubility or ionization ability of this compound. The radiation-induced hydrolysis, as one of the main processes resulting in loss of the extraction ability upon irradiation, is also typical for aliphatic amides, such as malonamides ${ }^{37}$ and diglycol amides. ${ }^{5}$

Samples irradiated in the presence of nitric acid contained a variety of degradation products similar to the case of ageing, including substance IV that was established for $6 \mathrm{M}$ nitric acid solution. In contrast to the samples that had no contact with nitric acid, there was no significant amount of hydrolysis products, which evidences about hydrolysis suppression by nitric acid under gamma-irradiation.

From these results, we can assume a scheme (Fig. 4) of the ligand (1) degradation occurring during irradiation and in contact with nitric acid.

Ligand (2). Mass spectra of samples of the ligand (2) in contact with nitric acid solutions revealed only two peaks at $\mathrm{m} / \mathrm{z}$ 650.47 and 672.45 , corresponding to the initial ligand (Table 6, compound $\mathbf{X}$ ) and its adduct with the sodium cation. All other peaks were almost at the noise level. So, any peaks that could be attributed to the products of nitration, nitrosation, or hydrolysis of the ligand were absent from the spectra.

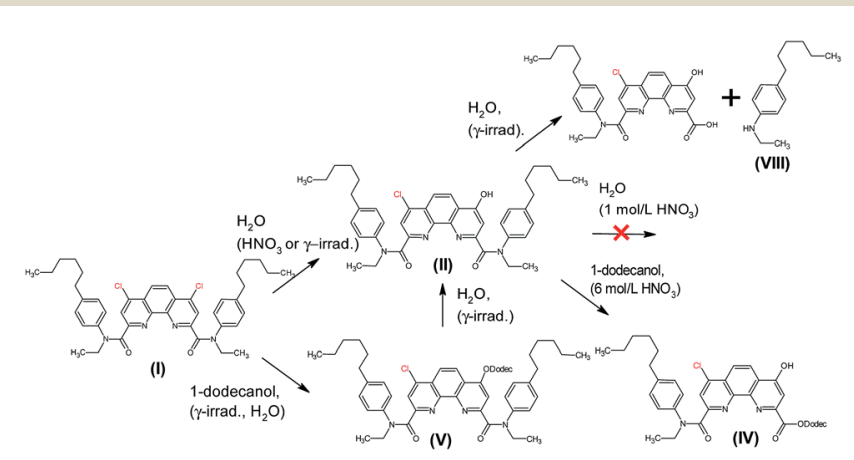

Fig. 4 The main processes occurring in the systems containing ligand (1) during gamma-irradiation and for ageing.

Table 6 Compounds detected in mass spectra of ligand (2) after irradiation in different conditions and the fragmentation products of molecular ion at $\mathrm{m} / \mathrm{z}$ 836.66. Fragmentation was done by collision induced dissociation (CID) at normalized collision energy (NCE) 40

$\begin{array}{lll}\text { No. Peak } m / z & \begin{array}{l}\text { Molecular formula and } \\ \text { monoisotopic mass }\end{array} \\ \mathbf{X I} \quad 650.47 & \mathrm{C}_{43} \mathrm{H}_{59} \mathrm{~N}_{3} \mathrm{O}_{2}, 649.4607 \\ \text { XII } \quad 866.38 & \mathrm{C}_{37} \mathrm{H}_{47} \mathrm{~N}_{3} \mathrm{O}_{2}, 565.3668 \\ \text { XIII } 836.66 & \mathrm{C}_{55} \mathrm{H}_{83} \mathrm{~N}_{3} \mathrm{O}_{2}, 817.6485\end{array}$


In the case of irradiated samples which did not contact with nitric acid solutions, the absolute intensity of the ligand peak decreased with accumulated dose. At the same time, the peak at $m / z 836.66$, corresponding to the product of ligand reaction with 1-dodecanol (XIII), increased with irradiation dose. Since the molecular mass of the product XIII is equal to the sum of ligand (2) and 1-dodecanol masses, the reaction occurred through the addition rather than the substitution. Most likely, a splitting of one lactam cycle occurred in this case.

In order to establish the structure of compound XIII, we performed fragmentation of the molecular ion corresponding to peak at 836.66. The resulting analysis of mass spectrum is shown in Table 6.

A singly charged ion peak at $m / z 818.66$ (XII) was the most abundant in the fragmentation spectrum. The mass difference between XII and XIII is 18, which corresponds to the splitting of one water molecule. This means that the starting compound (XIII) has a free hydroxyl group. This is consistent with the fact that 1-dodecanol was added in the form of an alpha-hydroxy radical. Fragmentation spectrum also contained a peak at 566.38 (XI) corresponding to splitting of the hexyl fragment from the initial ligand. All transformations that occurred with the ligand (2) are shown in Fig. 5.

Thus, no hydrolysis of the amide groups for the ligand (2) under the experimental conditions had been revealed, and the main process leading to a drop in the extraction efficiency is the interaction with 1-dodecanol. Using of more inert solvent, such as $m$-nitrobenzotrifluoride ${ }^{\mathbf{1 4}}$ widely used in the radiochemical industry, should increase the radiation resistance even in the absence of nitric acid.

\section{Theoretical calculations and their comparison with experimental results}

Ligand (1). As calculation of Fukui indices is based on computed partial charges condensed on atoms, its values depend on chosen computational method, basis set and molecule conformation. ${ }^{38}$ Here we consider only change of indices during protonation/deprotonation process as a chemical descriptor, in order to neglect all the mentioned factors. Moreover, we averaged indices for atoms in symmetry related

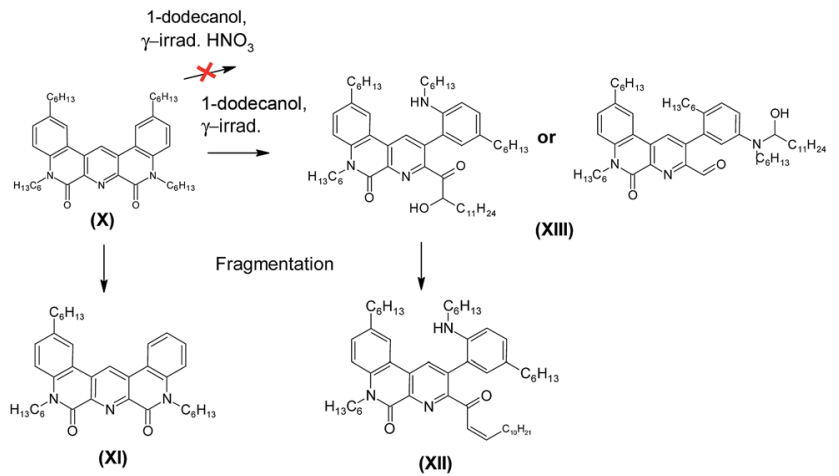

Fig. 5 Reactions occurring with the ligand (2) during gamma radiation, including fragmentation of the peak with $\mathrm{m} / \mathrm{z}=836.66$. positions of ligands (1) and (2), and protonated ligand (2) molecules to decrease its conformational dependence.

Fig. 6 and Table 7 show atoms with condensed indices values more than 0.02. Red lines stand for bonds with MBO less than 0.9 (Table 8). According to this, $\mathrm{C} 2$ and $\mathrm{C} 4$ (as well as symmetric $\mathrm{C}^{\prime}{ }^{\prime}$ and $\mathrm{C}^{\prime}$ ) atoms were the most reactive ones in phenantroline fragment. Indeed, we noticed chlorine substitution reactions with adding hydroxy- (in a case of ageing and irradiation) and alkoxy- (only for irradiation) groups to C4 atom. Also we found di-substituted products, but in a much smaller quantity. We may predict nitration process for $\mathrm{C} 2$ atom, but it is impossible to determine nitro-group position using MS method.

We also observed hydrolysis products as well as products of chlorine substitution, especially under irradiation. Hydrolysis corresponds attack on carbonyl carbon atom and one may predict relatively high values of radical and nucleophilic Fukui indices there. But according to our calculations the indices were negligibly small.

Ligand protonation leads to redistribution of electron density and change of Fukui indices. Radical index for $\mathrm{C} 4$ atom increased from 0.026 to 0.076 , while for $\mathrm{C} 2$ it decreased from 0.046 to 0.022 . As protonation was not symmetric in this case, such an activation occured only for $\mathrm{C} 4$ atom, while symmetric $\mathrm{C}^{\prime}$ ' became less active. It correlates with experimental data of chlorine substitution: it goes more intensively in a presence of nitric acid and it leads to the formation of mono-substituted products.

Besides, calculated activity increased for C5 atom the nearest bond was one of "weak bonds" according to our calculations. We may predict detachment of $p$-hexyl-phenyl fragment, as well as bond cleavage for $\mathrm{C} 1$ atom where the calculated situation was the same. We didn't find any products of $\mathrm{C}_{\text {arom }}-\mathrm{C}_{\text {aliph }}$ bond break, though such processes were described in literature for bipiridyl-bistriazines. ${ }^{39}$ In general, for both of ligands we observed "weakest" bonds in case of $\mathrm{C}_{\text {arom }}-\mathrm{C}_{\text {aliph }}$ bonds or $\mathrm{C}-\mathrm{N}$ bonds. Also according to our calculations protonation of ligands doesn't significantly affect on location and MBO values of the "weakest" bonds.

Ligand (2). The most reactive centers of ligand (2) (with symmetry averaging) are presented at Fig. 7 and Table 9. The only noticed reaction was 1-dodecanol addition in samples without nitric acid. We may suppose amine nitrogen atoms to be involved in that process.

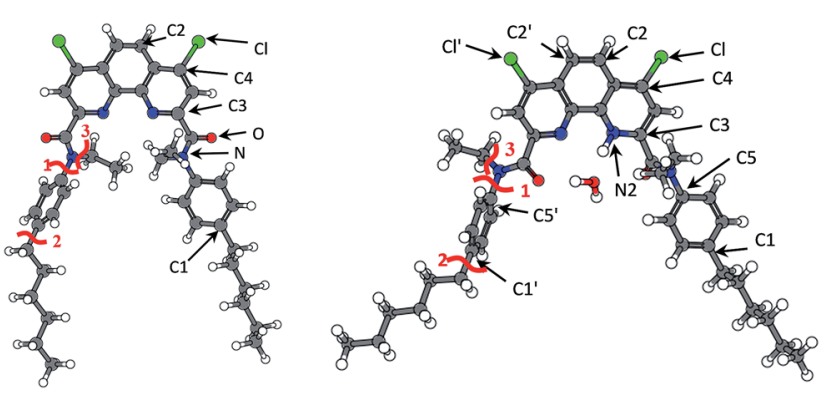

Fig. 6 Fukui indices for radical susceptibility (black arrows) and Mayer bond order (MBO, red lines) for (1) (left) and (1)_ $\mathrm{H}_{3} \mathrm{O}^{+}$(right). 
Table 7 Calculated condensed Fukui indices (with absolute value > 0.02 ) for ligand (1) and its protonated form

\begin{tabular}{|c|c|c|c|c|c|c|c|}
\hline \multicolumn{4}{|l|}{ (1) } & \multicolumn{4}{|l|}{ (1) $\_\mathrm{H}_{3} \mathrm{O}^{+}$} \\
\hline \multirow[b]{2}{*}{ Atom } & \multicolumn{3}{|c|}{ Fukui ind. } & \multirow[b]{2}{*}{ Atom } & \multicolumn{3}{|c|}{ Fukui ind. } \\
\hline & $\mathrm{R}$ & $\mathrm{E}$ & $\mathrm{N}$ & & $\mathrm{R}$ & $\mathrm{E}$ & $\mathrm{N}$ \\
\hline \multirow[t]{2}{*}{$\mathrm{C} 1$} & 0.050 & - & 0.091 & $\mathrm{C} 1$ & 0.048 & - & 0.076 \\
\hline & & & & $\mathrm{C} 1^{\prime}$ & 0.054 & - & 0.091 \\
\hline \multirow[t]{2}{*}{$\mathrm{C} 2$} & 0.046 & 0.076 & - & $\mathrm{C} 2$ & 0.022 & 0.032 & - \\
\hline & & & & $\mathrm{C} 2^{\prime}$ & 0.024 & 0.044 & - \\
\hline C3 & 0.041 & 0.097 & - & C3 & 0.050 & 0.11 & - \\
\hline \multirow[t]{3}{*}{$\mathrm{C} 4$} & 0.026 & 0.041 & - & $\mathrm{C} 4$ & 0.076 & 0.15 & - \\
\hline & & & & C5 & 0.031 & - & - \\
\hline & & & & $\mathrm{C}^{\prime}$ & 0.039 & - & 0.098 \\
\hline $\mathrm{N}$ & 0.038 & 0.025 & - & $\mathrm{N} 2$ & 0.043 & 0.087 & - \\
\hline $\mathrm{O}$ & 0.052 & 0.049 & - & $\mathrm{O}$ & 0.023 & 0.038 & - \\
\hline $\mathrm{C} 7$ & - & -0.025 & 0.046 & & & & \\
\hline C1amide & - & - & 0.002 & C1amide & - & -0.024 & 0.005 \\
\hline C2amide & - & - & - & C2amide & - & - & 0.009 \\
\hline
\end{tabular}

Table 8 The lowest MBO values for ligands (1) and (2) ligands and their hydronium complexes

\begin{tabular}{llll}
\hline & \multicolumn{2}{l}{ Bond no. } & \\
\cline { 2 - 4 } Ligand & 1 & 2 & 3 \\
\hline$(1)$ & 0.82 & 0.87 & 0.89 \\
$(1) \_\mathrm{H}_{3} \mathrm{O}^{+}$ & 0.78 & 0.86 & 0.89 \\
$(2)$ & 0.85 & 0.83 & - \\
$(2) \_\mathrm{H}_{3} \mathrm{O}^{+}$ & 0.89 & 0.89 & -
\end{tabular}

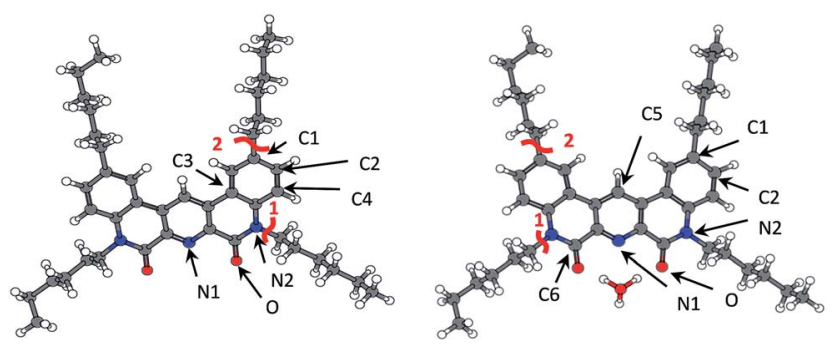

Fig. 7 Fukui indices for radical susceptibility (black arrows) and Mayer bond order (MBO, red lines) for ligand (2) and ligand (2) + hydronium complex (right).

We didn't observe any products of 1-dodecanol addition for samples with nitric acid. At the same time, our calculations showed a decrease of radical Fukui indices for heteroatoms in amide groups. Addition suppression might be also explained by sterical factor, cause large hydronium cation covers all possible directions of radical attack.

Also C5 Fukui index increased due to redistribution of electron density. But there were no reactions with this atom in our experiment, that can be probably explained by sterical problems. One can see hydrogen substitution at a similar atom in (2,6-bis(5-(2,2-dimethylpropyl)-1H-pyrazol-3-yl)pyridine) radiolysis. $^{34}$
Table 9 Calculated condensed Fukui indices (with absolute value > 0.02 ) for ligand (2) and its protonated form

\begin{tabular}{|c|c|c|c|c|c|c|c|}
\hline \multicolumn{4}{|l|}{ (2) } & \multicolumn{4}{|c|}{ (2) $\_\mathrm{H}_{3} \mathrm{O}^{+}$} \\
\hline \multirow[b]{2}{*}{ Atom } & \multicolumn{3}{|c|}{ Fukui ind. } & \multirow[b]{2}{*}{ Atom } & \multicolumn{3}{|c|}{ Fukui ind. } \\
\hline & $\mathrm{R}$ & $\mathrm{E}$ & $\mathrm{N}$ & & $\mathrm{R}$ & E & $\mathrm{N}$ \\
\hline C1 & 0.050 & - & 0.081 & $\mathrm{C} 1$ & 0.051 & - & 0.076 \\
\hline $\mathrm{C} 2$ & 0.039 & 0.058 & - & $\mathrm{C} 2$ & 0.037 & - & - \\
\hline C3 & 0.026 & - & 0.033 & C5 & 0.095 & - & - \\
\hline $\mathrm{C} 4$ & 0.022 & - & - & C6 & 0.021 & - & 0.035 \\
\hline $\mathrm{N} 1$ & 0.074 & 0.126 & - & $\mathrm{N} 1$ & 0.081 & 0.103 & - \\
\hline $\mathrm{N} 2$ & 0.043 & - & - & $\mathrm{N} 2$ & 0.027 & - & - \\
\hline $\mathrm{O}$ & 0.061 & 0.054 & - & $\mathrm{O}$ & 0.050 & 0.030 & - \\
\hline
\end{tabular}

Same as for ligand (1), the weakest bonds in the ligand (2) are $\mathrm{C}_{\text {arom }}-\mathrm{C}_{\text {aliph }}$ and $\mathrm{C}-\mathrm{N}$ (Table 8). Also, we didn't observe any bond breaking under gamma-irradiation. But analyzing ESI-MS spectra we noticed the peak at $\mathrm{m} / \mathrm{z}=566$, which corresponds to ligand (2) without hexyl group (structure XI in Table 5 and Fig. 6). So, predicted weak bond cleavage can take place in another conditions. But in a case of gamma-radiolysis, the main destructive factor is the interaction with radical products of solvent and water radiolysis.

\section{Conclusions}

We studied the ageing process of ligands (1) and (2) dissolved in the organic phase during the time up to 6 months. The process of external gamma irradiation was investigated up to absorbed doses of 200 and $500 \mathrm{kGy}$ for ligands (1) and (2), respectively. The samples were aged or irradiated with or without presence of nitric acid solutions.

Theoretical calculations of Fukui indices revealed the most reactive centers in extractant molecules taking part in the observed degradation processes. Under the irradiation conditions we didn't observe any predicted bond breakage, so we can affirm that interaction with solvent molecules fragments (radical or ionic) is a driving force of radiation degradation process.

We clearly illustrated and explained mechanism of ligands radioprotection in presence of acid solution. We also assume that change of a solvent can also increase radiolytic stability of ligands even in absence of nitric acid.

Nevertheless, we used only relative values of Fukui indices that doesn't allow to compare ligands reactivity. At the present moment, we leave quantitative assessment of radiolysis resistance for further investigations.

\section{Conflicts of interest}

There are no conflicts to declare.

\section{Acknowledgements}

The research is carried out using the equipment of the Center for collective use of HPC computing resources at Lomonosov 
Moscow State University. The reported study was supported by RFBR, research project No. 16-33-00491 мол_а.

\section{References}

1 C. J. Maher, Current headend technologies and future developments in the reprocessing of spent nuclear fuels, Elsevier Ltd., 2015.

2 M. Y. Alyapyshev, V. A. Babain and Y. A. Ustynyuk, Russ. Chem. Rev., 2016, 85, 943-961.

3 B. J. Mincher, G. Modolo and S. P. Mezyk, Solvent Extr. Ion Exch., 2009, 27, 579-606.

4 B. J. Mincher, G. Modolo and S. P. Mezyk, Solvent Extr. Ion Exch., 2010, 28, 415-436.

5 H. Galán, C. A. Zarzana, A. Wilden, A. Núñez, H. Schmidt, R. J. M. Egberink, A. Leoncini, J. Cobos, W. Verboom, G. Modolo, G. S. Groenewold and B. J. Mincher, Dalton Trans., 2015, 44, 18049-18056.

6 C. A. Zarzana, G. S. Groenewold, B. J. Mincher, S. P. Mezyk, A. Wilden, H. Schmidt, G. Modolo, J. F. Wishart and A. R. Cook, Solvent Extr. Ion Exch., 2015, 33, 431-447.

7 K. M. Roscioli-Johnson, C. A. Zarzana, G. S. Groenewold, B. J. Mincher, A. Wilden, H. Schmidt, G. Modolo and B. Santiago-Schübel, Solvent Extr. Ion Exch., 2016, 34, 439453.

8 T. Koubský and L. Kalvoda, J. Radioanal. Nucl. Chem., 2015, 304, 227-235.

9 T. Koubský, J. Fojtíková and L. Kalvoda, Prog. Nucl. Energy, 2017, 94, 208-215.

10 H. Galán, A. Núñez, A. G. Espartero, R. Sedano, A. Durana and J. de Mendoza, Procedia Chem., 2012, 7, 195-201.

11 M. Peräkylä and T. A. Pakkanen, J. Chem. Soc., Perkin Trans. 2, 1995, 1405-1410.

12 M. Y. Alyapyshev, V. A. Babain, L. I. Tkachenko, I. I. Eliseev, A. V. Didenko and M. L. Petrov, Solvent Extr. Ion Exch., 2011, 29, 619-636.

13 M. Y. Alyapyshev, V. A. Babain, L. I. Tkachenko, A. Paulenova, A. A. Popova and N. E. Borisova, Solvent Extr. Ion Exch., 2014, 32, 138-152.

14 M. Alyapyshev, J. Ashina, D. Dar'In, E. Kenf, D. Kirsanov, L. Tkachenko, A. Legin, G. Starova and V. Babain, RSC Adv., 2016, 6, 68642-68652.

15 J. L. Lapka, A. Paulenova, R. S. Herbst and J. D. Law, Sep. Sci. Technol., 2010, 45, 1706-1710.

16 E. A. Mowafy, Radiochim. Acta, 2007, 95, 539-545.

17 Y. A. Ustynyuk, N. E. Borisova, V. A. Babain, I. P. Gloriozov, A. Y. Manuilov, S. N. Kalmykov, M. Y. Alyapyshev,
L. I. Tkachenko, E. V. Kenf and N. A. Ustynyuk, Chem. Commun., 2015, 51, 7466-7469.

18 H. V. Lavrov, N. A. Ustynyuk, P. I. Matveev, I. P. Gloriozov, S. S. Zhokhov, M. Y. Alyapyshev, L. I. Tkachenko, I. G. Voronaev, V. A. Babain, S. N. Kalmykov and Y. A. Ustynyuk, Dalton Trans., 2017, 46, 10926-10934.

19 J. J. P. Stewart, J. Mol. Model., 2013, 19, 1-32.

20 J. J. P. Stewart, MOPAC2016, Stewart Computational Chemistry, Colorado Springs, CO, USA.

21 A. D. Becke, J. Chem. Phys., 1993, 98, 5648-5652.

22 P. J. Stephens, F. J. Devlin, C. F. Chabalowski and M. J. Frisch, J. Phys. Chem., 1994, 98, 11623-11627.

23 W. J. Hehre, R. Ditchfield and J. A. Pople, J. Chem. Phys., 1972, 56, 2257-2261.

24 F. Neese, Wiley Interdiscip. Rev.: Comput. Mol. Sci., 2012, 2, 73-78.

25 S. Grimme, J. Antony, S. Ehrlich and H. Krieg, J. Chem. Phys., 2010, 132, 154104.

26 I. Mayer, Chem. Phys. Lett., 1983, 97, 270-274.

27 I. Mayer, Theor. Chim. Acta, 1985, 67, 315-322.

28 I. Mayer, Int. J. Quantum Chem., 1984, 26, 151-154.

29 J. L. Gazquez and F. Mendez, J. Phys. Chem., 1994, 98, 45914593.

30 F. Mendez and J. L. Gazquez, J. Am. Chem. Soc., 1994, 116, 9298-9301.

31 A. Reed, R. Weinstock and F. Weinhold, J. Chem. Phys., 1985, 83, 735-746.

32 T. Y. Nikolaienko, L. A. Bulavin and D. M. Hovorun, Comput. Theor. Chem., 2014, 1050, 15-22.

33 A.-R. Allouche, J. Comput. Chem., 2011, 32, 174-182.

34 A. Wilden, G. Modolo, M. Hupert, B. Santiago-Schübel, E. Löfström-Engdahl, J. Halleröd, C. Ekberg, B. J. Mincher and S. P. Mezyk, Solvent Extr. Ion Exch., 2016, 34, 1-12.

35 B. J. Mincher, S. P. Mezyk, G. Elias, G. S. Groenewold, C. L. Riddle and L. G. Olson, Solvent Extr. Ion Exch., 2013, 31, 715-730.

36 R. Stephenson and J. Stuart, J. Chem. Eng. Data, 1986, 31, 5670.

37 L. Berthon, J. M. Morel, N. Zorz, C. Nicol, H. Virelizier and C. Madic, Sep. Sci. Technol., 2001, 36, 709-728.

38 T. Y. Nikolaienko, L. A. Bulavin and D. M. Hovorun, RSC Adv., 2016, 6, 74785-74796.

39 A. Fermvik, L. Berthon, C. Ekberg, S. Englund, T. Retegan and N. Zorz, Dalton Trans., 2009, 32, 6421-6430. 